\title{
Meta-analysis of the impact of intervention versus symptom-driven management in asymptomatic severe aortic stenosis
}

\author{
Wei Yao Lim, ${ }^{1}$ Anantharaman Ramasamy, ${ }^{1}$ Guy Lloyd ${ }^{1,2,3}$ Sanjeev Bhattacharyyaa ${ }^{1,2,3}$
}

${ }^{1}$ Echocardiography Laboratory, Barts Heart Centre, St Bartholomew's Hospital, London, UK

${ }^{2}$ Valvular Heart Disease Clinic, Barts Heart Centre, St Bartholomew's Hospital, London, UK

${ }^{3}$ Institute of Cardiovascular Science, University College London, London, UK

Correspondence to Dr Sanjeev Bhattacharyya, Echocardiography Laboratory \& Valvular Heart Disease Clinic, Barts Heart Centre, St Bartholomew's Hospital, Institute of Cardiovascular Sciences, UCL, London EC1 7AB, UK; Sanjeev. Bhattacharyya@bartshealth. nhs.uk

Received 18 April 2016 Revised 28 July 2016 Accepted 1 August 2016 Published Online First 18 August 2016

\section{SLinked}

http://dx.doi.org/10.1136/ heartjnl-2016-310399

\section{CrossMark}

To cite: Lim WY, Ramasamy A, Lloyd $\mathrm{G}$, et al. Heart 2017;103:268-272.

\section{ABSTRACT \\ Objective The management of patients with} asymptomatic, severe aortic stenosis (AS) is controversial. We performed a meta-analysis to examine the impact on outcomes of aortic valve replacement (AVR) in patients with severe asymptomatic AS versus a symptom-driven intervention approach.

Methods A search for studies that examined the outcomes of AVR and management of asymptomatic severe AS was performed. We examined the end points of all-cause mortality, cardiac mortality and sudden cardiac death. Our analysis compared early AVR in asymptomatic patients with a symptom-driven AVR approach (excluding symptomatic patients who did not undergo AVR).

Results Four observational studies were identified with a total of 1300 patients. There was significant heterogeneity between studies $\left(1^{2}=72 \%\right)$. Using a random-effects model, there was a trend towards reduced overall mortality in patients undergoing early AVR compared with a symptom-driven AVR approach (OR $0.54,95 \% \mathrm{Cl} 0.26$ to $1.12, p=0.1$ ). There was no significant reduction in cardiac mortality or sudden death ( $O R 0.78, p=0.85$, and $O R \quad 0.34, p=0.25$, respectively). Conclusions Although there was a trend towards reduced overall mortality when comparing early AVR in patients with asymptomatic, severe AS to a symptomdriven AVR approach, there was no significant difference in cardiac mortality or sudden death. An individual approach focusing on individual risk stratification and operative mortality is required until more robust, randomised trial data are available.

\section{INTRODUCTION}

Aortic stenosis (AS) affects $>10 \%$ of the population $>65$ years. ${ }^{1}$ Aortic valve replacement (AVR) is recommended for symptomatic patients with severe AS as the prognosis without intervention is dismal. ${ }^{2}$ The management of asymptomatic patients with severe AS is controversial. Observational studies have shown the annual risk of sudden death is about $1 \%$ per year. ${ }^{3}$ The risk of AVR will vary depending on patient comorbidities, although it has been falling. ${ }^{4}$ Current guidelines do not recommend intervention in asymptomatic patients unless they are in certain high-risk groups such as those with very severe asymptomatic AS or those with haemodynamic changes on exercise. ${ }^{5}$

There have been several observational studies looking at the outcome of patients with asymptomatic AS. ${ }^{6-11}$ Two recent studies have suggested benefit from early AVR in asymptomatic patients. ${ }^{67}$ Interpretation of these studies and translation into clinical practice is difficult as a proportion of patients in the observation/symptom-driven arm often did not undergo AVR despite developing symptoms. No randomised controlled trials have been conducted to test this hypothesis.

We conducted a meta-analysis of these studies to determine whether early AVR compared with symptom-driven AVR is beneficial in patients with severe asymptomatic AS.

\section{METHODS}

\section{Search strategy}

An electronic search on the Web of Knowledge comprising Medline, Web of Science, BIOSIS and Data Citation Index was performed. The search was performed and included studies published until July 2016. The search terms used were 'asymptomatic', 'AS', 'outcomes', 'AVR' and 'surgery'. No language constraints were applied to the search.

\section{Study selection}

Two independent reviewers (WYL and AR) screened all articles to identify potentially eligible studies. The full text of these potentially eligible studies was then evaluated to determine the eligibility of the study for the meta-analysis. The reference lists of these articles were also reviewed. All 'reviews', 'case reports', 'letters', 'meetings' and 'abstracts' were excluded. Studies selected were required to examine the outcome of patients with asymptomatic severe AS and include both asymptomatic patients treated with an early AVR and asymptomatic patients treated with a symptombased AVR strategy (referral for AVR when patients became symptomatic only). Only studies with survival data were included. Studies would have to define severe AS as an aortic valve area $\leq 1 \mathrm{~cm}^{2}$ or peak aortic velocity $\geq 4 \mathrm{~m} / \mathrm{s}$. Where multiple studies were conducted on the same population of patients, only one study was included. A Preferred Reporting Items for Systematic Reviews and Meta-Analyses flow chart detailing the selection process is included in figure 1 .

\section{End points}

The primary end point was all-cause mortality. Secondary end points were cardiac mortality and sudden cardiac death. Cardiac mortality was defined as death due to heart failure, myocardial infarction, sudden death, aortic valve 
Figure 1 Preferred Reporting Items for Systematic Reviews and Meta-Analyses flow chart detailing selection criteria for meta-analysis. AR, aortic regurgitation; $A S$, aortic stenosis; AVR, aortic valve replacement; LV, left ventricle.
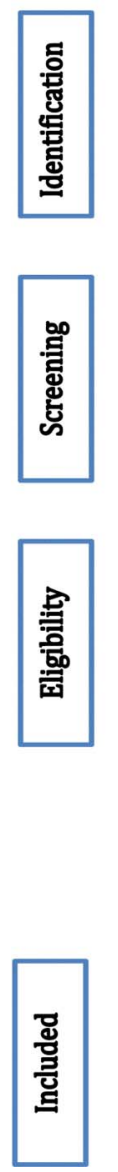

procedure-related death, infective endocarditis, stroke and aortic/peripheral vascular disease.

The analysis compared the outcomes of patients undergoing early AVR to patients within a symptom-driven AVR protocol (patients either underwent AVR after developing symptoms or remained asymptomatic). Patients who developed symptoms but did not undergo AVR were excluded.

\section{Statistics}

OR and 95\% CI was calculated for each end point using both a random effects and fixed model. Early AVR was considered as the experimental group and therefore an OR $<1$ favours early AVR. Statistical heterogeneity was assessed and quantified using the Cochran Q test and the $\mathrm{I}^{2}$ statistic. $\mathrm{p}$ Values $<0.05$ were considered significant. All values were two-sided. Statistical analysis was performed using Review Manager 5.3. Publication bias was analysed using funnel plots and Egger test. This analysis was performed using Stats Direct, V.3.0.124.

\section{RESULTS}

A total of four published papers met the search criteria (table 1). All were observational studies. A total of 1300 patients were enrolled in the studies. Overall, there was significant heterogeneity between studies $\mathrm{I}^{2}=72 \%$. Overall, there was no significant publication bias $(\mathrm{p}=0.71)$.

\section{Effect of AVR on overall mortality}

All four studies included data on overall mortality. In a random-effects model, early AVR in patients with asymptomatic severe AS was associated a trend towards reduced overall

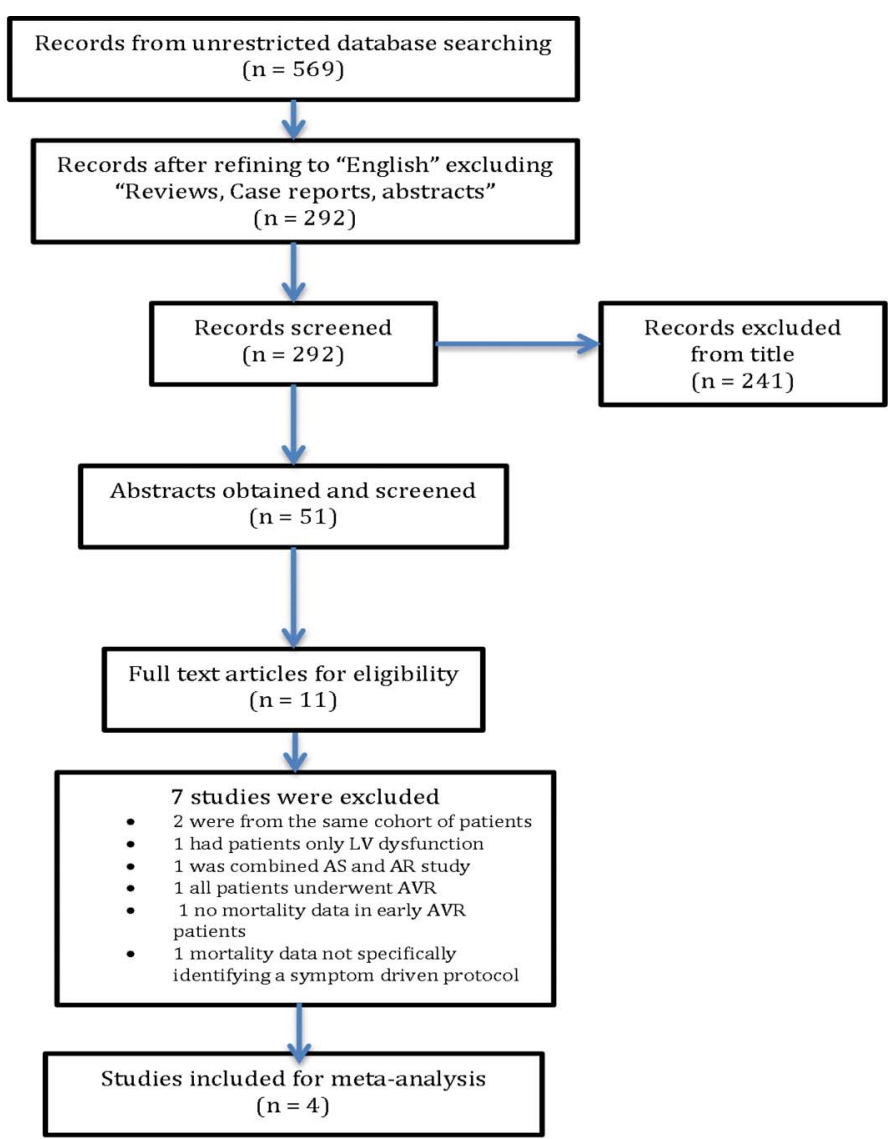

mortality compared with a symptom-driven AVR approach (OR $0.54,95 \%$ CI 0.26 to $1.12, p=0.1$ ) (figure $2 \mathrm{~A}$ ). Using a fixed-effects model, early AVR was associated with significantly lower mortality compared with symptom-driven AVR (OR 0.58, $95 \%$ CI 0.43 to $0.77, \mathrm{p}=0.0002$ ) (figure $2 \mathrm{~B}$ ).

\section{Effect of AVR on cardiac mortality}

Three studies (838 patients) included data on cardiac mortality. There was no significant difference in cardiac mortality between patients undergoing early AVR and a symptom-driven AVR approach using a random-effects model (OR 0.78, 95\% CI 0.06 to $10.12, \mathrm{p}=0.85$ ) (figure $3 \mathrm{~A}$ ). There was a trend towards reduced cardiac mortality using a fixed-effects model (OR 0.64, $95 \%$ CI 0.38 to $1.08, \mathrm{p}=0.10$ ) (figure $3 \mathrm{~B}$ ).

\section{Effect of AVR on sudden cardiac death}

Three studies (838 patients) included data on cardiac sudden death (91 events). Using a random-effects model, there was no significant difference in mortality due to sudden death between patients undergoing early AVR and those treated with a symptom-driven AVR approach (OR $0.34,95 \%$ CI 0.06 to $2.11, \mathrm{p}=0.25$ ). However, using a fixed-effects model early AVR was associated with a trend towards a reduction in risk of sudden death compared with a symptom-driven approach (OR $0.44,95 \%$ CI 0.20 to $1.00, p=0.05$ ).

\section{DISCUSSION}

A key dilemma is whether the risk of AVR in a patient with asymptomatic severe AS outweighs the risk of sudden death if a patient was to be followed up using a symptom-guided 
Table 1 Summary of studies included in meta-analysis

\begin{tabular}{|c|c|c|c|c|c|c|c|c|}
\hline $\begin{array}{l}\text { Study and publication } \\
\text { year }\end{array}$ & $\begin{array}{l}\text { Study } \\
\text { period }\end{array}$ & $\begin{array}{l}\text { Number of } \\
\text { patients }\end{array}$ & Follow-up (months) & Age (years) & $\begin{array}{l}\text { Male } \\
\text { n (\%) }\end{array}$ & $\begin{array}{l}\text { Hypertension } \\
(\%)\end{array}$ & $\begin{array}{l}\text { Diabetes } \\
\mathrm{n}(\%)\end{array}$ & $\begin{array}{l}\text { Coronary artery } \\
\text { disease } \\
\text { n (\%) }\end{array}$ \\
\hline Pellikka et al $(2005)^{9}$ & 1984-1995 & 622 & Mean $64.8 \pm 48$ & $72 \pm 11$ & $384(62 \%)$ & $272(44 \%)$ & $71(11 \%)$ & Excluded \\
\hline Kang et al $(2010)^{7}$ & 1996-2006 & 197 & Median 50 & $63 \pm 12$ & $99(50 \%)$ & $76(39 \%)$ & $20(10 \%)$ & Excluded \\
\hline Heuvelman et al (2012) ${ }^{10}$ & 2006-2009 & 59 & Fixed follow-up 24 & $69.9(61.6-76.4)$ & $45(76 \%)$ & $29(49 \%)$ & $13(22 \%)$ & $\begin{array}{l}\text { Myocardial infarction } \\
5(8 \%) \\
\text { Coronary artery bypass } \\
\text { graft } 2(3 \%)\end{array}$ \\
\hline Taniguchi $(2015)^{6}$ & 2003-2011 & 582 & Median $45.4(35-56.6)^{*}$ & $\begin{array}{l}71.6 \pm 8.7 \dagger \\
73.1 \pm 9.3 \ddagger\end{array}$ & $250(43 \%)$ & $375(64 \%)$ & $125(22 \%)$ & $135(23 \%)$ \\
\hline
\end{tabular}

approach. Two recent, non-randomised, observational studies have suggested early AVR is associated with a mortality benefit ${ }^{6} 7$ compared with patients treated 'conventionally'. A recent meta-analysis confirmed these findings. ${ }^{12}$ However, in all of these studies a large proportion of patients who developed symptoms did not undergo AVR, illustrating a potential bias in non-randomised studies. Therefore, we analysed our data comparing early AVR in asymptomatic patients to a symptom-driven AVR approach. Given the heterogeneity between studies (different time periods, hospitals, follow-up periods, patient demographics), the true effect may vary from study to study and therefore a random-effects model may be preferable. Using the random-effects model, the data suggest early AVR may be associated with reduced overall mortality compared with a symptom-based AVR approach, but there does not seem to be a significant effect on cardiac mortality and sudden death. In view of this, we do not believe the data are sufficient to change clinical practice until more robust, randomised data are available.
The risk stratification of patients with asymptomatic severe AS is complex. Patients with moderate or severe aortic valve calcification and a rapid increase in aortic jet velocity $(\geq 0.3 \mathrm{~m} / \mathrm{s}$ increase in aortic velocity per year), very severe AS (aortic velocity $\geq 5 \mathrm{~m} / \mathrm{s}$ or mean pressure gradient $\geq 60 \mathrm{~mm} \mathrm{Hg}$ ) and those with adverse haemodynamic changes during exercise (fall in blood pressure during exercise) have a high likelihood of cardiac events in the next couple of years. ${ }^{11} 13{ }^{14}$ International guidelines recommend consideration of early AVR in these groups, but do not recommend intervention in all asymptomatic patients. ${ }^{5}$ Recently, the concept of adverse risk relating to the severity of AS has been extended to aortic valve area. Maréchaux et al ${ }^{15}$ demonstrated the risk of mortality in asymptomatic patients increased as aortic valve area reduced. Patients with an aortic valve area $\leq 0.6 \mathrm{~cm}^{2}$ had a threefold increased risk of mortality compared with those with an aortic valve area of $>0.6 \mathrm{~cm}^{2}$. There are several other proposed markers of prognosis in severe asymptomatic AS. ${ }^{3}$ However, many of these use
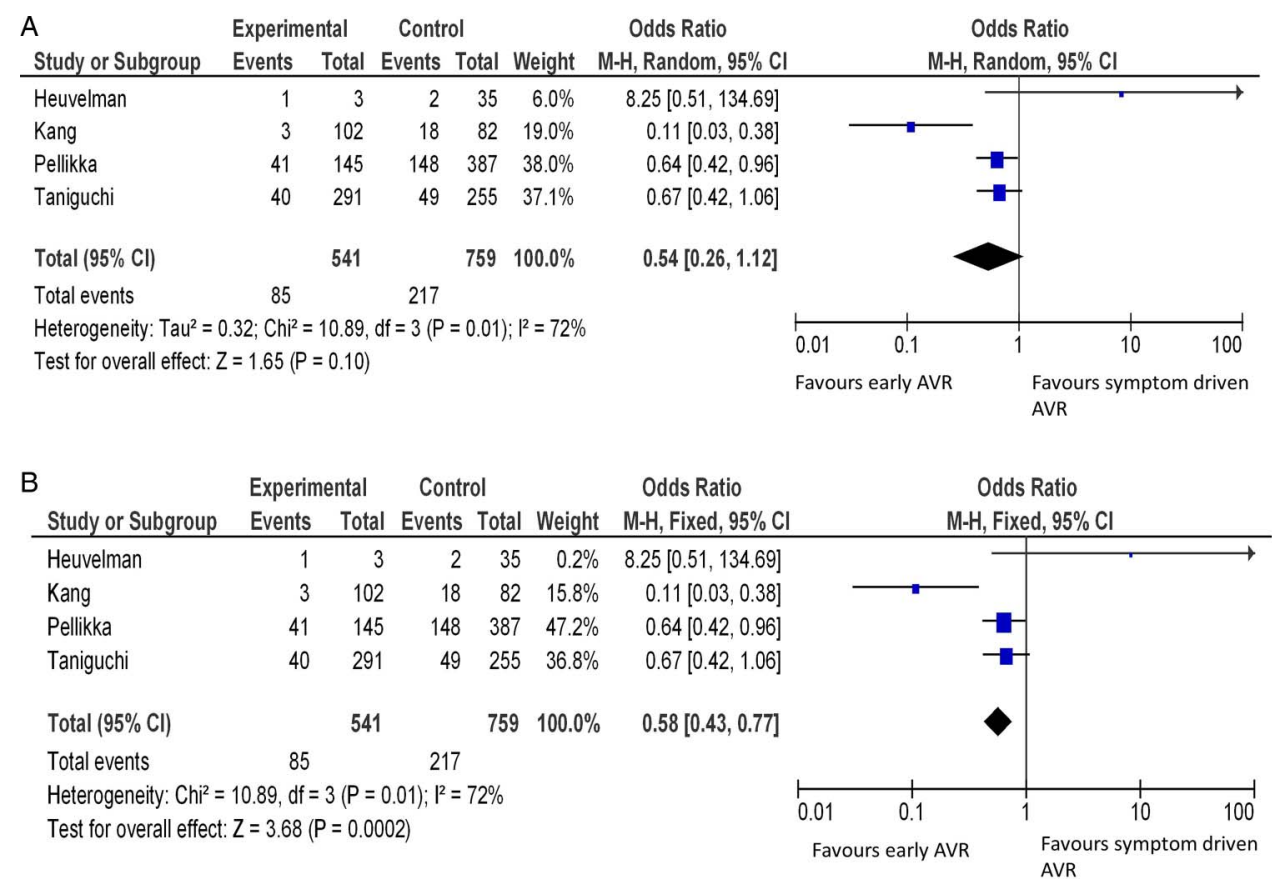

Figure 2 (A) Random-effects model comparing all-cause mortality between patients with asymptomatic severe aortic stenosis undergoing early aortic valve replacement (AVR) and patients on a symptom-driven AVR protocol. (B) Fixed-effects model comparing all-cause mortality between patients with asymptomatic severe aortic stenosis undergoing early AVR and patients on a symptom-driven AVR protocol. 

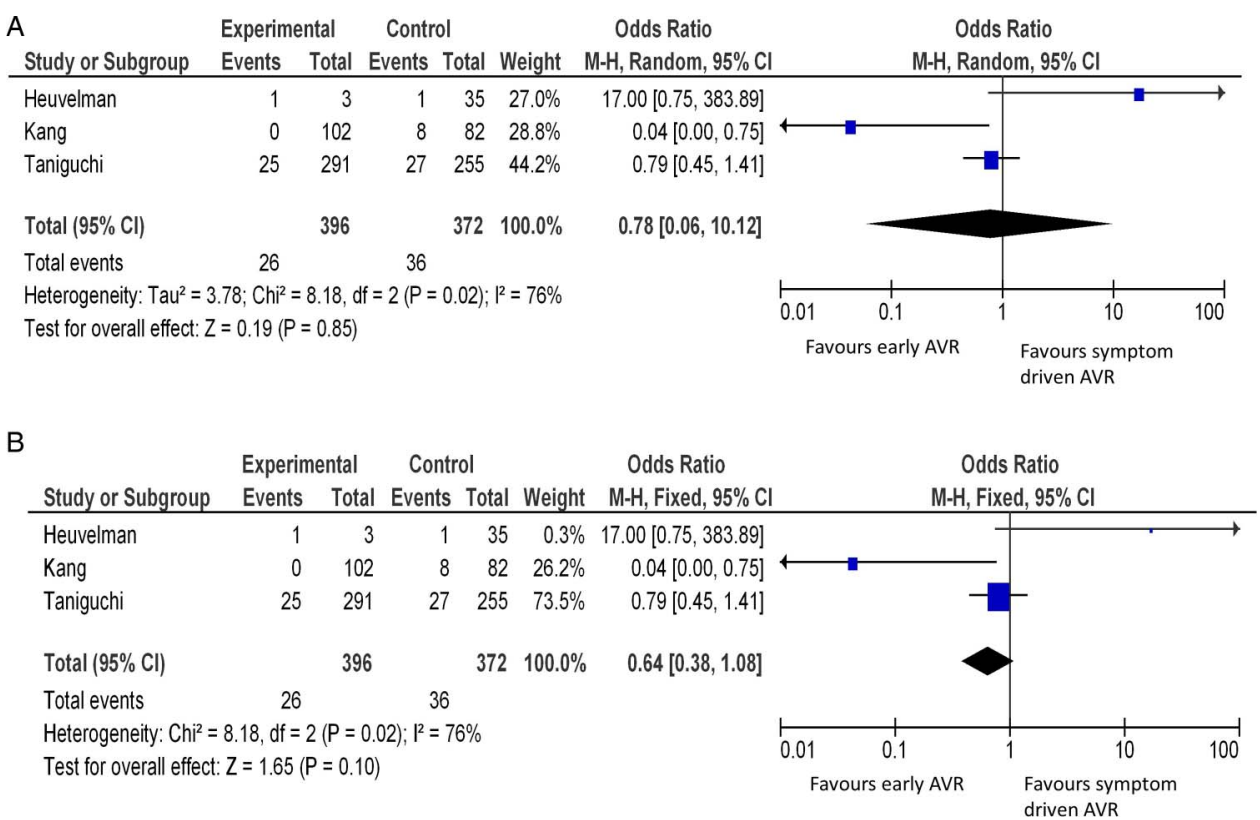

Figure 3 (A) Random-effects model comparing cardiac mortality between patients with asymptomatic severe aortic stenosis undergoing early aortic valve replacement (AVR) and patients on a symptom-driven AVR protocol. (B) Fixed-effects model comparing cardiac mortality between patients with asymptomatic severe aortic stenosis undergoing early AVR and patients on a symptom-driven AVR protocol.

a combined end point of AVR and death. As AVR is usually performed on development of symptoms, these markers may be predictive of symptom development rather than pure mortality.

The risk of sudden death per year varies between the population of patients studied. A rate of sudden death from $0.2 \%$ to $13.3 \%$ per year has been reported. ${ }^{3}$ The variation is likely to represent differences in both individual patient risk and the method of follow-up. One of the drawbacks of waiting for symptoms is the difficulty in ensuring patient adherence to prompt reporting of symptoms and follow-up. It also possible some patients become higher risk for AVR at the time of becoming symptomatic if they develop complications such as heart failure. In addition, assessing symptom status can be difficult. A recent community-based study in patients with a mean age of 74 years showed symptoms of dyspnoea on exertion, angina and near syncope are not specific for severe AS and occur in 39\% of patients with mild AS. ${ }^{16}$ This highlights the difficulty in symptom assessment in elderly patients with impaired mobility and comorbidities. The risk of AVR will depend on both shortterm perioperative risk (dependent on age and comorbidities) and longer-term risk (stroke, valve reoperation, endocarditis, bleeding) ${ }^{17}$ There has been a decline in the mortality of AVR over the past decade, particularly in centres that offer transcatheter aortic valve implantation. ${ }^{18}$ Therefore, an individualised approach taking account of these factors is required for an individual patient decision.

A limitation of our analysis is all the studies analysed were observational and are subject to possible selection bias. The outcome data were not uniform between studies, and therefore not all studies could be included in each subanalysis and HRs could not be calculated. There was significant heterogeneity between studies, which spanned three decades with differing follow-up duration. The definition of severe AS varied between studies, and, consequently, in some patients with subtypes of AS, that is, pseudo-severe AS may have been included. Furthermore, there was no standardised assessment of symptom status (exercise testing) or identification of adherence to follow-up protocols.
A randomised controlled trial in asymptomatic severe AS is currently underway, and this would add important data that would help define management strategies. ${ }^{19}$ We would advocate waiting for the results of this trial prior to making firm recommendations for the management of this patient group.

Our analysis of the published data suggests early AVR may be associated with reduced overall mortality compared with a symptom-based AVR approach, but there does not seem to be a significant effect on cardiac mortality and sudden death. In view of this, an individual approach focusing on individual risk stratification and operative mortality is required until more robust data are available.

\section{Key messages}

\section{What is already known on this subject?}

The optimal management of patients with asymptomatic severe aortic stenosis (AS) is uncertain. Guidelines do not recommend routine early aortic valve replacement (AVR) in asymptomatic patients unless certain high-risk features are present. However, several recent studies have suggested benefit from early AVR in asymptomatic patients.

\section{What might this study add?}

This is the first study to examine the outcome of early AVR in patients with asymptomatic severe AS to a symptom driven AVR approach. Although there was a trend towards reduced overall mortality from early AVR compared with a symptom-driven AVR approach, the published data are not robust enough to support this as generalised approach at present.

How might this impact on clinical practice? An individual approach focusing on individual risk stratification and operative mortality is required until randomised data are available. 
Contributors $S B$ conceived the idea for the manuscript. WYL and AR performed the literature search. WL, AR and SB performed the analysis. WYL, AR, SB and GL drafted the manuscript. All authors approved the final manuscript.

Competing interests None declared.

Provenance and peer review Not commissioned; externally peer reviewed.

\section{REFERENCES}

1 Nkomo VT, Gardin JM, Skelton TN, et al. Burden of valvular heart diseases: a population-based study. Lancet 2006;368:1005-11.

2 Schwarz F, Baumann P, Manthey J, et al. The effect of aortic valve replacement on survival. Circulation 1982;66:1105-10.

3 Bhattacharyya S, Hayward C, Pepper JR, et al. Risk stratification in asymptomatic severe aortic stenosis: a critical review. Eur Heart J 2012;33:2377-87.

4 O'Brien SM, Shahian DM, Filardo G, et al. The society of thoracic surgeons 2008 cardiac surgery risk models: part 2-isolated valve surgery. Ann Thorac Surg 2009;88:S23-42.

5 Nishimura RA, Otto CM, Bonow RO, et al. CC/AHA Task Force Members. 2014 AHA/ACC guideline for the management of patients with valvular heart disease: a report of the American College of Cardiology/American Heart Association Task Force on Practice Guidelines. Circulation 2014;129:e521-643.

6 Taniguchi T, Morimoto T, Shiomi H, et al. CURRENT AS Registry Investigators. Initial surgical versus conservative strategies in patients with asymptomatic severe aortic stenosis. J Am Coll Cardiol 2015;66:2827-38.

7 Kang DH, Park SJ, Rim JH, et al. Early surgery versus conventional treatment in asymptomatic very severe aortic stenosis. Circulation 2010;121:1502-9.

8 Pai RG, Kapoor N, Bansal RC, et al. Malignant natural history of asymptomatic severe aortic stenosis: benefit of aortic valve replacement. Ann Thorac Surg 2006;82:2116-22.

9 Pellikka PA, Sarano ME, Nishimura RA, et al. Outcome of 622 adults with asymptomatic hemodynamically significant aortic stenosis during prolonged follow-up. Circulation 2005;111:3290-5.
10 Heuvelman HJ, van Geldorp MW, Kappetein AP, et al. Clinical course of patients diagnosed with severe aortic stenosis in the Rotterdam area: insights from the AVARIJN study. Neth Heart J 2012;20:487-93.

11 Rosenhek R, Binder T, Porenta G, et al. Predictors of outcome in severe, asymptomatic aortic stenosis. N Engl J Med 2000;343:611-17.

12 Généreux P, Stone GW, O'Gara PT, et al. Natural history, diagnostic approaches, and therapeutic strategies for patients with asymptomatic severe aortic stenosis. J Am Coll Cardiol 2016;67:2263-88.

13 Amato MCM, Moffa PJ, Werner KE, et al. Treatment decision in asymptomatic aortic valve stenosis: role of exercise testing. Heart 2001;86:381-6.

14 Rosenhek R, Zilberszac R, Schemper $M$, et al. Natural history of very severe aortic stenosis. Circulation 2010;121:151-6.

15 Maréchaux S, Ringle A, Rusinaru D, et al. Prognostic value of aortic valve area by doppler echocardiography in patients with severe asymptomatic aortic stenosis. J Am Heart Assoc 2016;5:e003146.

16 Malouf J, Le Tourneau T, Pellikka P, et al. Aortic valve stenosis in community medical practice: determinants of outcome and implications for aortic valve replacement. J Thorac Cardiovasc Surg 2012;144:1421-7.

17 Brennan JM, Edwards FH, Zhao Y, et al, DECIDE AVR (Developing Evidence to Inform Decisions about Effectiveness-Aortic Valve Replacement) Research Team. Long-term safety and effectiveness of mechanical versus biologic aortic valve prostheses in older patients: results from the Society of Thoracic Surgeons Adult Cardiac Surgery National Database. Circulation 2013; 127:1647-55.

18 Brennan JM, Holmes DR, Sherwood MW, et al. The association of transcatheter aortic valve replacement availability and hospital aortic valve replacement volume and mortality in the United States. Ann Thorac Surg 2014;98:2016-22.

19 Banovic M, lung B, Bartunek J, et al. Rationale and design of the Aortic Valve replAcemenT versus conservative treatment in Asymptomatic seveRe aortic stenosis (AVATAR trial): a randomized multicenter controlled event-driven trial. Am Heart J 2016;174:147-53. 